\title{
SOLAR ACTIVITY OBSERVED IN X-RAYS \\ AND THE EUV FROM OSO 7
}

\author{
ROGER J. THOMAS \\ Laboratory for Solar Physics and Astrophysics, NASA-Goddard Space Flight Center, \\ Greenbelt, Md., U.S.A.
}

\begin{abstract}
Since 1971 the Goddard X-ray and EUV spectroheliograph aboard OSO 7 has been measuring the spatial distribution and time-variations of localized temperature and density features in solar active centers and flares. In some cases the sizes, shapes, orientations and locations of emitting plasmas at temperatures ranging from $10^{4} \mathrm{~K}(\mathrm{H} \alpha)$ to as high as $2 \times 10^{7} \mathrm{~K}$ (Fe xxv) have now been measured simultaneously. Our observations of active regions are consistent with the coronal structure being made up of nested systems of arches with footpoints in areas of opposite magnetic polarity. Temperatures seem to increase for arches nearer the center and also towards the top of any given magnetic arch, the innermost loops having the highest temperature gradients. There is also some evidence for electric current flow along such loops. Radiative cooling is significant for the region's hot central core which therefore must be maintained by a more or less continuous injection of energy.

A nested arch structure is also indicated for XUV flares of the two-component type, which likewise may require continuous energy input since conduction cooling should otherwise be very rapid. Multiple spikes during the impulsive phase seem to represent the consecutive triggering of different sources within the region and may occur outside of any detectable pre-existing coronal feature. Comparison of spatial distributions at several wavelengths during various stages of flare events provides information on interactions between the wide range of atmospheric levels involved. We have evidence for polarization of about $20 \%$ in a number of X-ray bursts, continuing throughout the decay phase. At least for some flares, our measurements seem to contradict the model of electron beams being radially injected into the chromosphere.
\end{abstract}

\section{Introduction}

The Goddard X-Ray and EUV spectroheliograph launched aboard OSO 7 on 29 September 1971 was designed to investigate a wide range of solar phenomena at wavelengths emitted by the corona and upper chromosphere. I will limit myself here to a discussion of just some of the results we have recently obtained on active regions and flares, using principally the spectroheliograms made simultaneously at several individual wavelengths between 1.7 and $400 \AA$ with a spatial resolution of $20^{\prime \prime} \times 20^{\prime \prime}$ or better. In addition, I will report on the initial analysis of our X-ray polarimeter measurements and the bearing they have on current flare theories. The instrument itself has already been described by Underwood and Neupert (1974).

\section{Active Regions}

Prior to the advent of observations from space, total solar eclipses and artificial eclipses achieved with ground-based coronagraphs provided the only opportunities for recording the form and structure of the high temperature corona. These observations, made with the Sun's disk occulted, provided valuable information on characteristic structures such as streamers and coronal condensations. However, the fundamental limitation that the underlying solar atmosphere could not be simultaneously recorded 
except at the limb made it difficult to associate coronal forms with more commonly observed photospheric and chromospheric features.

Now, results from X-ray and EUV telescopes carried above the Earth's atmosphere on rockets, satellites, and ATM have shown that much of the structure of the inner corona is in the form of loops, occurring either as connections between areas of opposite magnetic polarity within a given bipolar region or as higher arches connecting seemingly independent active regions (e.g., Vaiana et al., 1968). It has also become possible to measure directly the physical characteristics of these coronal condensations on the disk, thus providing a solid basis for an attack on the critical problem of determining exactly how they are formed and maintained. For example, using the Harvard spectroheliograph on OSO 4, Noyes et al. (1970) found that active regions are characterized on the average by greater electron pressure (by a factor of 5), higher coronal temperature $\left(2.5 \times 10^{6} \mathrm{~K}\right)$, and greater conductive flux back to the chromosphere (by a factor of 5) over that observed in the undisturbed corona. However, the obvious importance of fine-scale structure recently emphasized by the magnificent ATM observations presents a clear challenge to existing, highly simplified models of these coronal enhancements.

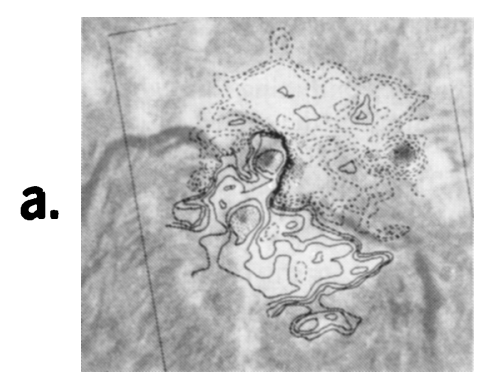

\section{$H$-alpha at 14:45UT}

\section{a. Photospheric Field at 16:19 UT}

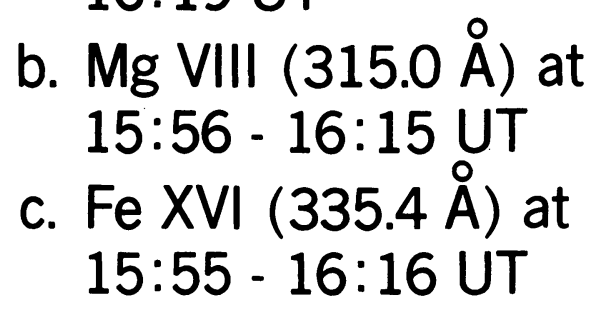

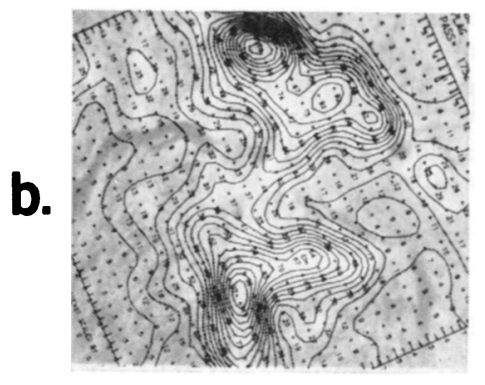

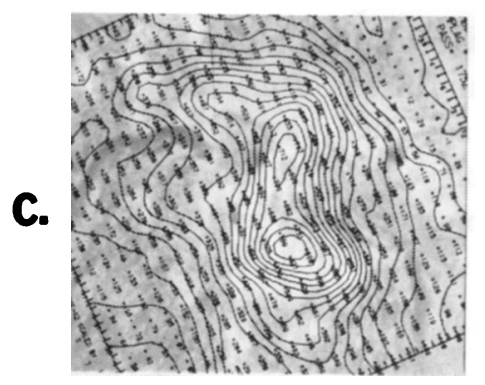

Fig. 1. Comparison of the photospheric magnetic field measured by Sacramento Peak Observatory with the EUV emission observed by OSO 7 for an active region at $15^{\circ} \mathrm{S}, 13^{\circ} \mathrm{E}$ on 1972, January 19.

Each map is overlayed on an $\mathrm{H} \times$ photograph of the region and covers an area $5^{\prime}$ on a side. 
During the $2 \frac{1}{2}$ yr lifetime of OSO 7, we have observed a vast number of active regions in all stages of development. I have selected one to describe in some detail, a dynamic flaring region that crossed the disk in January 1972, because it typifies many of the characteristics we have seen in other regions of this type.

Figure 1 shows the EUV emission of Mg VIII and Fe XVI from this region in the form of isophote contours overlayed on an $\mathrm{H} \alpha$ photograph taken at the Sacramento Peak Observatory. The photospheric magnetic field as measured by Rust (1974), is also indicated, with the neutral line running approximately east-west bisecting the region. The principal EUV structure is made up of a pair of sources, one on each side of the neutral line and therefore overlying areas of opposite magnetic polarity. In the transition zone and lower corona, however, numerous secondary enhancements appear, not all of which are associated with bright $\mathrm{H} \alpha$ plage, as can be seen in the $\mathrm{Mg}$ vIII map of Figure 1.

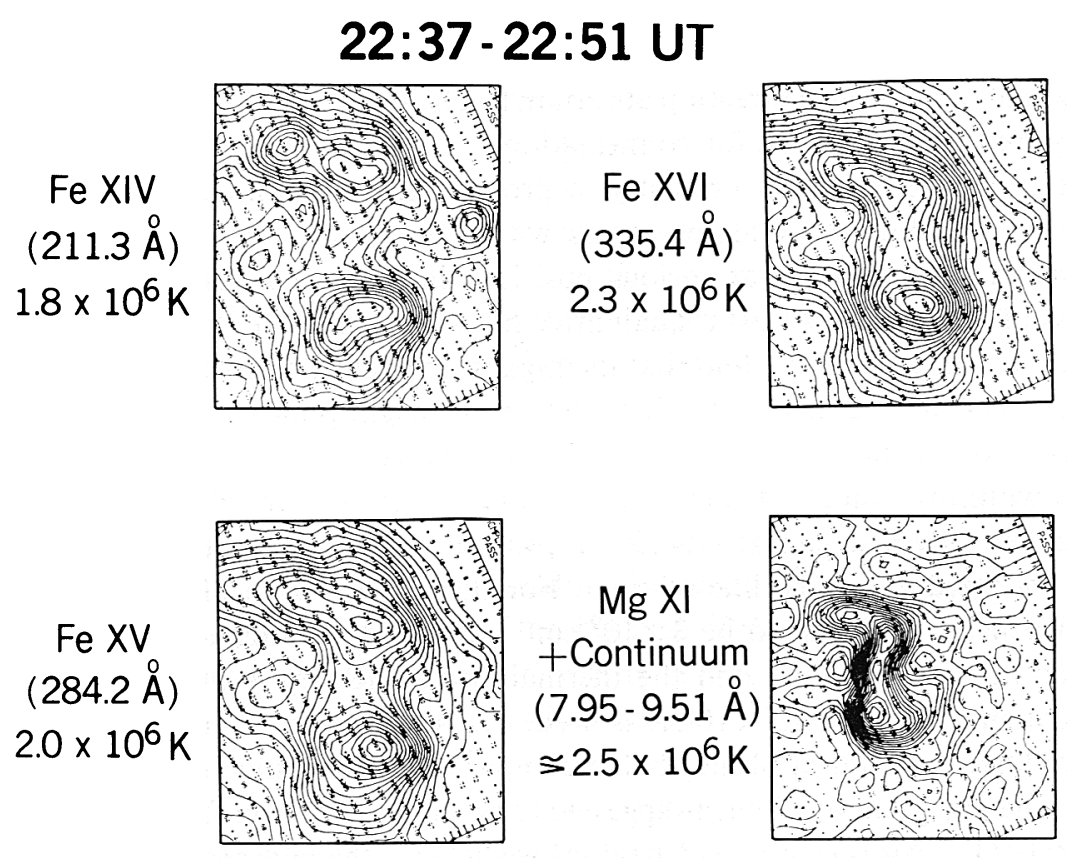

Fig. 2. Spatial distribution of EUV and X-ray emission arranged in a temperature sequence for the active region at $15^{\circ} \mathrm{S}, 9^{\circ} \mathrm{E}$ on 1972 , January 19 . The temperature given is that at which the contribution function for that line has its maximum value. Maps are roughly $3.7 \times 4.2^{\prime}$ in area. North is at the top in this and the preceding figure.

In Figure 2, several EUV maps of the region are arranged in a sequence of increasing temperature of emission, from Fe XIV at $1.8 \times 10^{6} \mathrm{~K}$ to $\mathrm{Mg}$ XI at $2.5 \times 10^{6} \mathrm{~K}$. It should be noted that the temperature given is not that at which the ion population is greatest, but rather the temperature at which the contribution function has its maximum, a more appropriate value as pointed out by Jordan (1975). At elevated coronal temperatures, the structure is seen to simplify, becoming much less contorted, and 
often takes on a linear form with bright $\mathrm{H} \alpha$ plage appearing near the extremities of the coronal emission. At the highest temperatures, it becomes very compact, giving the appearance of a hot central core reported by earlier observers such as Blake et al. (1964) and Pounds and Russell (1966).

The bridge of EUV emission between the two principal sources also seems to change its orientation with increasing electron temperature, for example rotating from a northwest-southeast direction to a directly north-south orientation in the $\mathrm{Mg}$ VIII and Fe XVI maps of Figure 1. This may be evidence for the existence of non-potential magnetic fields, and therefore electric currents, in the coronal enhancement overlying the plage. Nakagawa (1974, private communication) has calculated possible coronal field configurations for the region satisfying force-free field conditions and using a variety of assumptions about the direction and magnitude of the electric current flow. Although there are difficulties with the interpretation of such calculations, preliminary comparisons with our EUV observations suggest that various regimes of temperature in the corona may correspond to different strengths of the electric current, and that the flow may even reverse direction at certain levels. However, this tentative conclusion will require further analysis for confirmation.

Another area of research presently in progress involves an attempt to determine the spatial distribution of temperatures within active regions. Using ratios of the $\mathrm{Fe} \mathrm{XV}$ to Fe XVI intensities at various positions in maps such as those of Figure 2, it is possible to obtain at least a qualitative measure of how the coronal temperature varies across the region. We find that the highest temperature in an active region does not seem to occur at either of the Fe XV or Fe XVI bright points, but rather may occur at the location of the relatively compact X-ray source.

Now, within the limits of our spatial resolution, it is a straightforward matter to estimate the physical characteristics of just this small, highest-temperature EUV feature averaged along the line of sight. For the active region of January 1972, the electron density turns out to be $3 \times 10^{9} \mathrm{~cm}^{-3}$, based on a thickness of $14000 \mathrm{~km}$ as measured at the west limb, and the thermal energy density is therefore $3 \mathrm{erg} \mathrm{cm}^{-3}$ if a peak temperature of $2.7 \times 10^{6} \mathrm{~K}$ is used. For such a feature, it can be shown that radiative losses are not at all negligible compared to the conductive loss rates, contrary to what has sometimes been suggested to be the case for non-flaring active regions (Tucker, 1973). This is especially true if, as we believe, the high temperature feature is at the top of a magnetic arch where conduction will be somewhat inhibited. In fact, based on the loss rates calculated by Cox and Tucker (1969), the entire kinetic energy of this source should be radiated away in about $6000 \mathrm{~s}$, or less than two hours. Since the enhancement actually persists for many days, energy must be more or less continuously supplied in some way at the rate of roughly $2 \times 10^{-3} \mathrm{erg} \mathrm{cm}^{-3} \mathrm{~s}^{-1}$ throughout its lifetime. A similar though much less stringent conclusion was reached by Reidy et al. (1968) who found that the radiation cooling time of an active region could be as short as a half-day.

Our observations seem consistent with the following general picture. The coronal enhancement overlying an active region appears to be made up of a nested set of 
arches or loops, with footpoints in regions of opposite magnetic polarity. The outermost arches are coolest, while those nearer the center are hotter. But even along a given field line there is a temperature gradient such that the highest temperature occurs at the highest point of the magnetic arch. This temperature gradient is smallest for the cool, outer loops, and becomes significantly greater for the loops near the center. Such a picture appears to be quite compatible with the Skylab and rocket results already presented at this symposium.

\section{Flares}

Turning now to the question of flares, I think it is necessary to emphasize that this term may actually cover a number of fundamentally different phenomena, each presumably having its own energy conversion mechanism (see, for example, TandbergHanssen, 1973). Thus, when using results from different flares, one must be careful to consider only events of the same basic type. A similar difficulty arises in regard to the XUV bursts that accompany flares. De Jager (1965) was the first to suggest that X-ray bursts be divided into two classes, which are presently referred to as impulsive and gradual bursts. Often, a single event will contain both of these components. It is not yet clear to what extent such two-component bursts represent an additional unique class of events, or merely a combination of the two previously mentioned types.

I will restrict myself here to just the type which exhibits both components, a typical example of which is shown in Figure 3 (taken from Frost, 1969). The impulsive phase of such a burst consists of one or more extremely rapid spikes and normally lasts for only about two minutes. These spikes invariably occur during the rise of the associated gradual component, which itself may have the appearance of high-frequency fluctuations superimposed on a much more gradual rise and fall. Kane (1969) and others have shown that the impulsive component usually dominates at higher energies because of its harder spectrum. For that reason, and because of the rapid time variation, this component is almost certain to be non-thermal in character.

The situation is not so clear for the gradual component. Although it shows many thermal properties, there are several indications that non-thermal processes are still taking place during this phase. One of the most striking is the discovery from the New Hampshire experiment on OSO 7 that nuclear reactions are occurring in a flare for more than ten minutes (Chupp et al., 1973), that is, well after the impulsive burst. Another is the hardening of the spectrum above $12 \mathrm{keV}$ that Kahler et al. (1970) and others have seen throughout the gradual phase of some flares.

The close correspondence between the hard X-ray and microwave bursts is quite evident from Figure 3. In addition, Donnelly $(1967,1969)$ has demonstrated that variations in broad-band EUV emission are also very similar to those in the hard $\mathrm{X}$-ray burst, at least during the impulsive phase. Using the spectral resolution of the Harvard OSO experiments, Donnelly et al. (1973) later concluded that the EUV emission formed at temperatures up to a million degrees exhibited this impulsive behavior. 


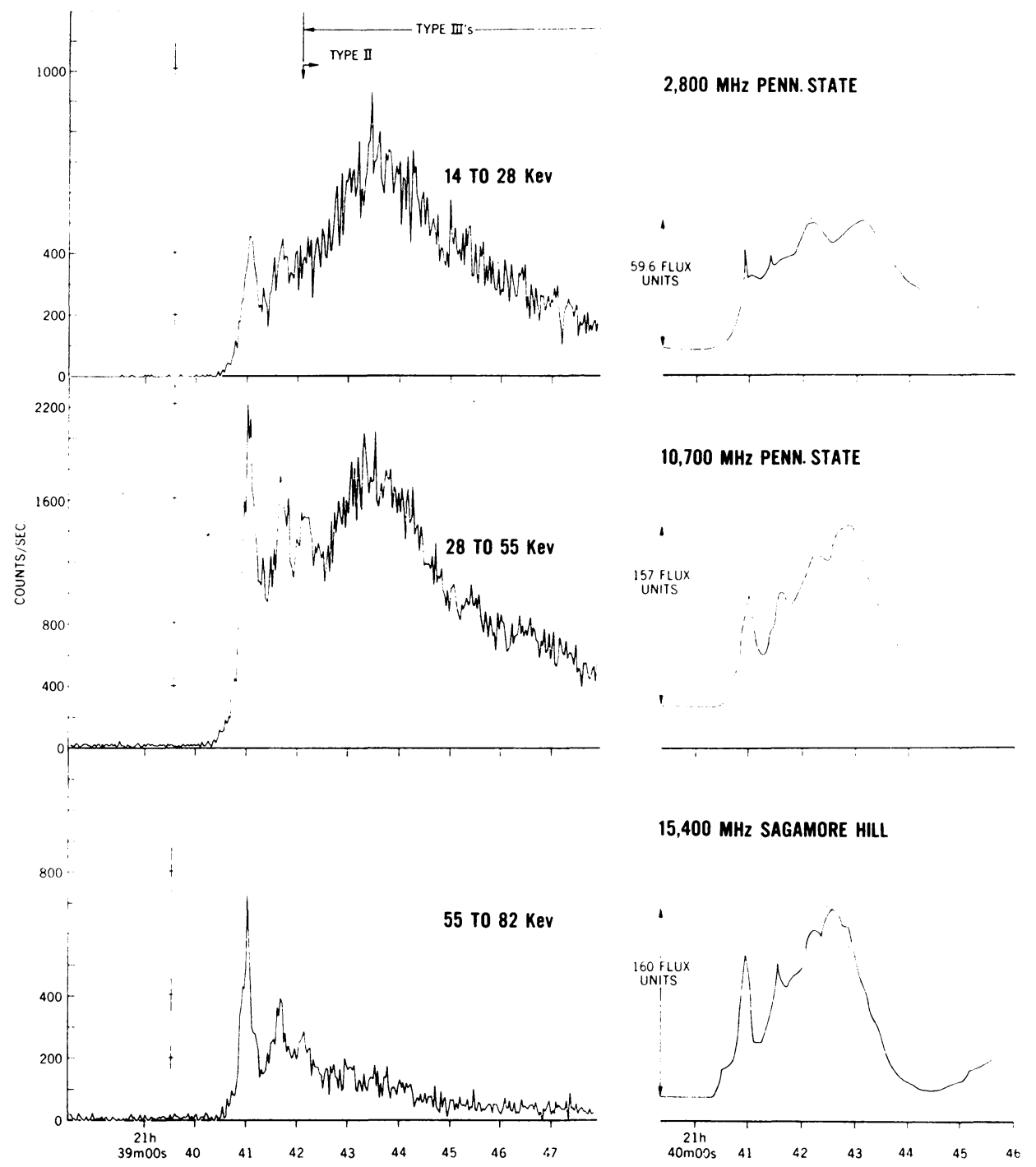

Fig. 3. A two-component solar burst on 1969, March 1 observed in hard X-rays by OSO 5 and at several radio frequencies by Penn. State and Sagamore Hill. The three leading impulsive spikes are followed by a component with a more gradual envelope and a distinctly different spectral shape. Taken from Frost (1969).

On the basis of X-ray measurements from OGO-5, Kane and Donnelly (1971) suggested that the EUV emission is produced thermally in a region heated by collisional losses of the non-thermal electrons responsible for the impulsive $\mathrm{X}$-ray and microwave bursts. They believe this heating must occur at densities above $10^{12} \mathrm{~cm}^{-3}$, that is, in the chromosphere. Such a result fits in very nicely with the discovery by Vorpahl and Zirin (1970) that impulsive bursts are associated with the chromospheric brightening of small kernels of $\mathrm{H} \alpha$ emission. 


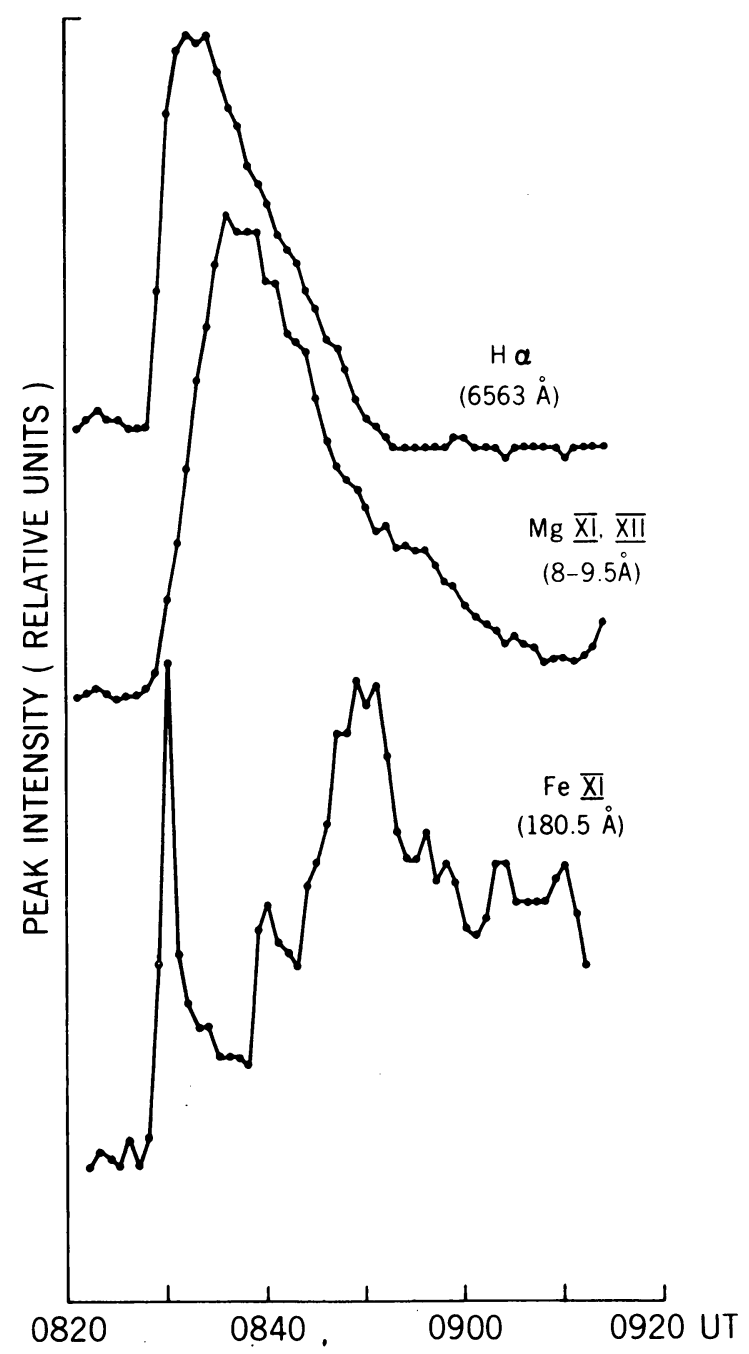

Fig. 4. Time history of the peak intensity of a flare on 1972, February 13 observed by OSO 7 at three wavelengths in the visible ( $\mathrm{H} \alpha)$, soft X-rays (MgXI, XII), and EUV (FexI).

The exact relation between the impulsive and gradual phases of this type of event is very much unknown, at least to me. From the University of California, San Diego experiment on OSO 7, Datlowe and Hudson (1975) have been able to show that the total energy contained in the electron beam responsible for the impulsive burst is adequate to account for the energy in the X-ray emitting plasma during the gradual phase, under certain assumptions. But whether or not the electron beam really is the only source remains an open question.

Now let me discuss a few of our Goddard OSO 7 flare observations that bear on some of these points. Figure 4 shows the time variations of a flare's peak intensity at three different wavelengths: $\mathrm{H} \alpha$, soft $\mathrm{X}$-rays, and an EUV line. The coronal X-ray 
emission of Mg XI and XII which is formed at about $9 \times 10^{6} \mathrm{~K}$ and the chromospheric $\mathrm{H} \alpha$ radiation both have just a simple rise and decay. On the other hand, the Fe XI emission at $1.2 \times 10^{6} \mathrm{~K}$ shows a great deal of structure, with an initial impulsive phase and then a rather complex gradual phase. At the instant of the impulsive burst, there is an abrupt rise in both $\mathrm{H} \alpha$ and $\mathrm{X}$-rays. With the one-minute time resolution of our observations, the impulsive EUV burst seems to be a single spike. But higher resolution data, such as from the New Hampshire X-ray monitor on OSO 7, indicate there were at least two spikes and perhaps even more during this interval.

\section{FLARE IMPULSIVE PHASE}

\section{FEB 1972 OSO-7}

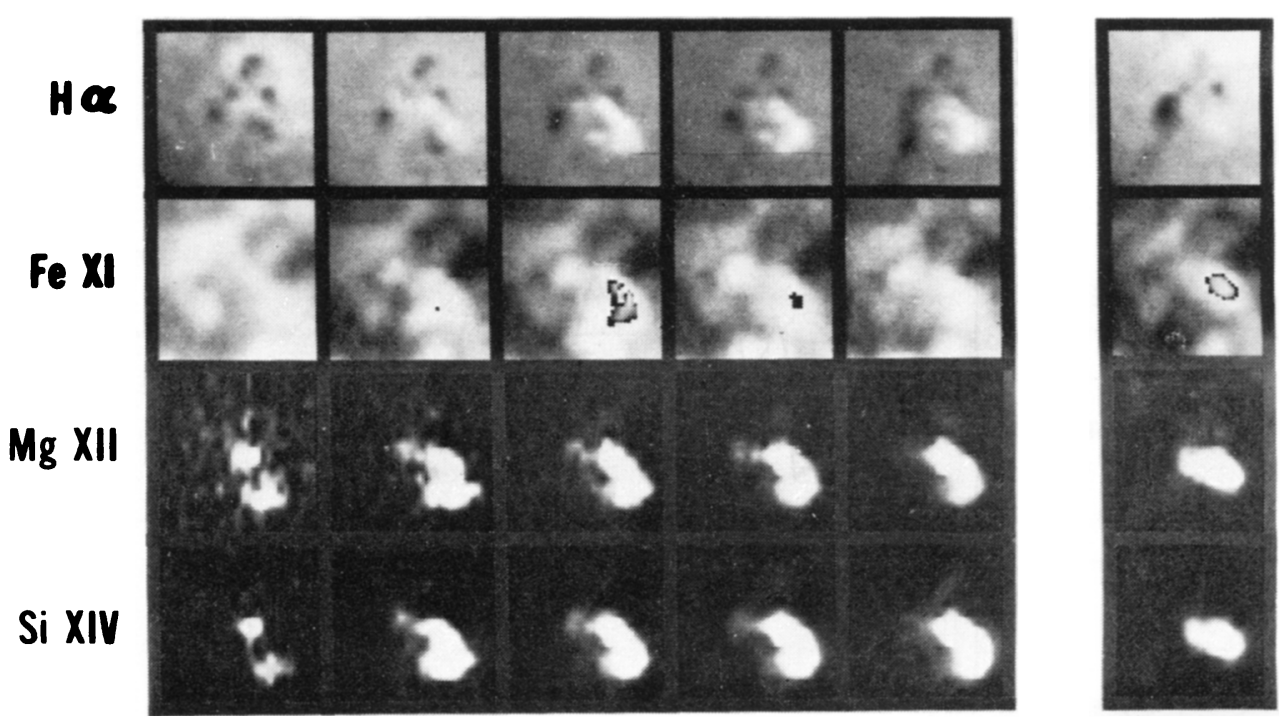

Fig. 5. - Time sequence from left to right of OSO 7 spatial maps in a grey-scale representation for the flare shown in Figure 4. From top to bottom are maps $5^{\prime}$ square in $\mathrm{H} \alpha, \mathrm{Fe} \mathrm{XI}, \mathrm{Mg} \mathrm{XI}-\mathrm{XII}$, and Si XIII-XIV. The sequence on the left covers the impulsive phase at roughly one-minute intervals. The set on the right were all made at the peak of the gradual component. Dark markings in the center of some Fe XI maps are due to computer overflow and actually represent the very brightest locations.

Each curve in Figure 4 gives the intensity of the flare's brightest point as a function of time, regardless of where its spatial location might be. But our EUV maps during the gradual phase show that there were actually two different locations that brightened and faded in an apparently independent manner, one peaking around 0840 UT and the other at 0850 UT. This independent behavior of different points seems to be typical of the gradual component formed in the transition zone and lower corona. It unfortunately makes the interpretation of spectra taken without spatial resolution very difficult, since the radiation may well be a combination from two distinct sources, one brightening while the other fades. 
We also found for this particular event that the impulsive EUV emission came from at least two different locations. Figure 5 is a time sequence from left to right of our spatial maps in a grey-scale representation. The top set are $\mathrm{H} \alpha$ maps, the second row are $\mathrm{Fe} \mathrm{XI}$, and the bottom two rows are maps in two soft X-ray bands. Incidently, these $\mathrm{H} \alpha$ observations were made from OSO 7, in fact through the same entrance aperture as the EUV, so there is no uncertainty whatsoever in the spatial correspondence with the other EUV maps. The sequence on the left covers the impulsive phase at roughly one-minute intervals, while the set on the right were all made at $0850 \mathrm{UT}$, the peak of the gradual component. Our computer overflowed for the very brightest Fe XI points, and these appear as odd dark markings in the center of some of the maps.

The dark speck in the second Fe XI map is the site of the initial impulsive burst. One minute later, the peak EUV emission appears in a different location, some $30000 \mathrm{~km}$ away. Apparently, multiple spikes do not represent the re-energizing of the same feature, but rather the consecutive triggering of different sources within the region.

Note that the $\mathrm{H} \alpha$ flare starts in a well-defined kernel seen in the second map of the top row of Figure 5. It is obvious, however, that this $\mathrm{H} \alpha$ kernel is not at all in the same location as the simultaneous EUV spike. The only way I can see to reconcile this observation with the otherwise attractive hypothesis of Kane and Donnelly (1971) is if the non-thermal electron beam entered the chromosphere at a very large slantangle. But this would seriously conflict with the radial-beam model suggested by the work of De Jager and Kundu (1963) that many believe is correct.

Another puzzling aspect is the fact that the impulsive emission seen in the third Fe XI map of Figure 5 is coming from a much larger region than the gradual source shown on the far right. If the total flux we measure can be used to indicate relative size (which seems reasonable since the peak intensities were almost identical), the volume of the impulsive source is three times that of the gradual source. Perhaps the impulsive phase involves a large number of separate electron beams scattered over a wide area producing a 'shot-gun' effect, whereas the heating during the gradual phase occurs in a more compact region.

As can be seen in the bottom two rows of Figure 5, the coronal X-ray enhancement, which was a double source before the flare, appears as a single elongated feature whose main axis seems to rotate during the event. I would assume that this rotation corresponds to a re-alignment of the coronal magnetic field configuration during the flare. If the flaring plasma cools by expansion, we see no evidence of it in our maps. In fact, the XUV sources may actually appear to contract as they fade.

Figure 6 shows the intensity variations at two EUV wavelengths of the brightest point in another flare, an event recently analyzed by Neupert et al. (1974). Although it does have a gradual phase, the burst is clearly dominated by the impulsive component in this case. The radiation at $295 \AA$ in the lower curve is almost entirely continuum and scattered light, principally from $\mathrm{H}$ I and $\mathrm{He}$ II, and so is characteristic of chromospheric temperatures. The top curve, however, is mainly Fe XIV, which radiates at about $1.8 \times 10^{6} \mathrm{~K}$. This extends the impulsive behavior to much higher temperatures than those reported by Donnelly et al. (1973). 


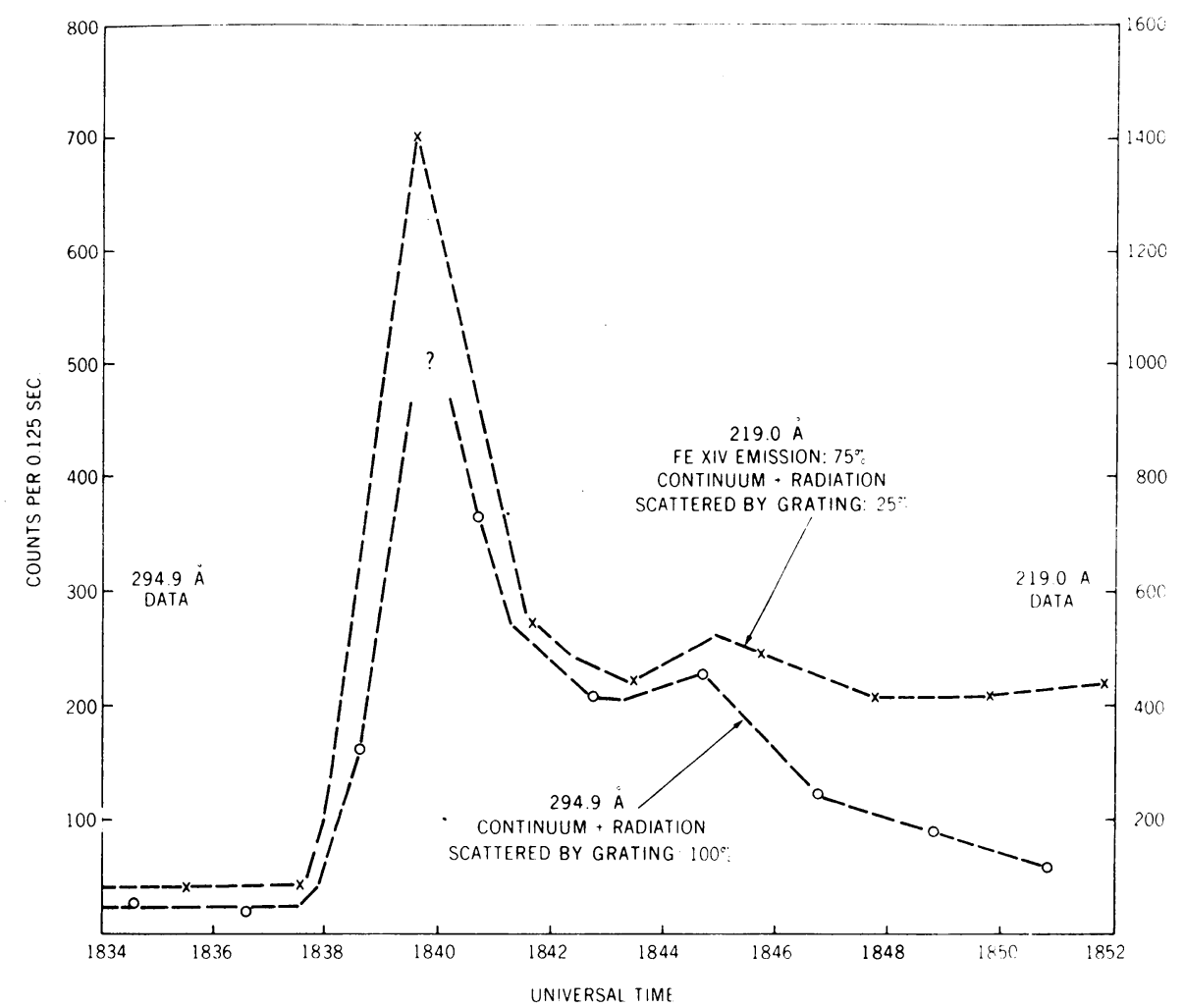

Fig. 6. The intensity of the brightest point in a flare on 1972, August 2 at two EUV wavelengths. The impulsive phase between 1838 and 1842 UT clearly dominates the following gradual component in this event. Figures 6, 7, and 8 are taken from Neupert et al. (1974).

Figure 7 shows the spatial distribution at these two wavelengths, as well as that of Fe XVII which radiates at about $3 \times 10^{6} \mathrm{~K}$. The maps on the right are during the impulsive burst, those on the left were made just prior to the event. The XUV enhancements before the flare are associated with areas of the active region that were bright in $\mathrm{H} \alpha$. But the flare emission is from a distinctly different location, some $50000 \mathrm{~km}$ away from the main pre-flare enhancement. Clearly, the flare does not originate in any pre-existing coronal feature, at least none that we can detect. In fact, one can show that simply heating or compressing the pre-existing coronal material at this location cannot account for the strength of the emission measured during the flare itself. We believe this means that the XUV-emitting flare plasma originated as a result of the heating of chromospheric, or perhaps even photospheric, material. For other flares, the situation is not always as clear as it was in this case. But I have not yet seen any flare in our OSO 7 data where the impulsive burst occurred at the brightest point of a pre-existing coronal feature.

Three spectroheliograms made during the gradual phase of the previous flare are shown in Figure 8, each superimposed on the same off-band $\mathrm{H} \alpha$ photograph from the Lockheed Solar Observatory. In addition to the chromospheric radiation at $295 \AA$ 


\section{9Å (CHROMOSPHERIC RADIATION)}
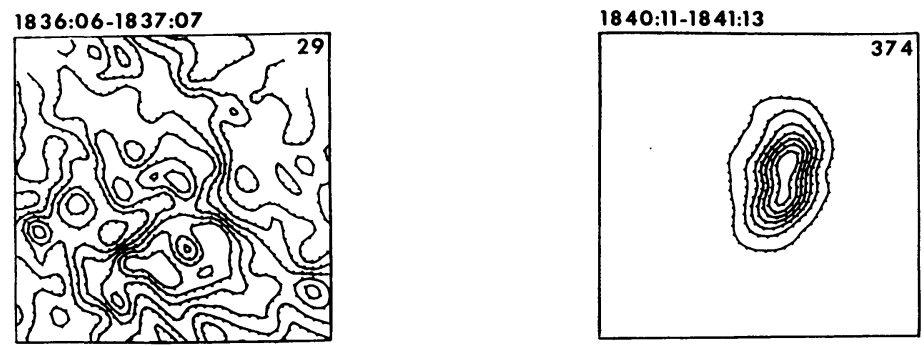

\section{$219.0 \AA$ (Fe XIV)}

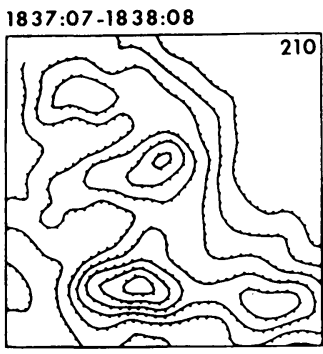

10

4 arc $\min$

$(=168,000 \mathrm{~km})$

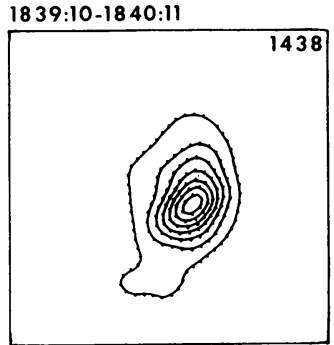

$14.50-15.90 \AA ̊ 丿$ (Fe XVII)

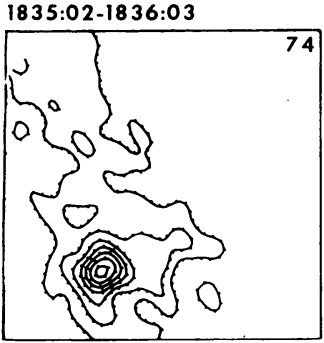

$1841: 13-1842: 14$

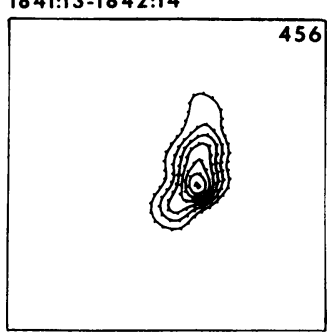

Fig. 7. Spatial maps of EUV and X-ray distributions observed by OSO 7 during (right) and just prior to (left) the impulsive phase of the flare shown in Figure 6. Isophote contours are scaled to one-eighth of the observed peak count which is indicated in the upper right corner of each map. Note that only weak EUV and no measurable soft X-ray emission emanated from the site of the flare before the event began.

(map a), we have isophotes of X-ray emission formed at about $9 \times 10^{6} \mathrm{~K}$ (map b) and at around $30 \times 10^{6} \mathrm{~K}$ (map c). All three sources are quite elongated, as is the $\mathrm{H} \alpha$ flare. However, only the features at $10 \times 10^{6} \mathrm{~K}$ or less are exactly coincident with the $\mathrm{H} \alpha$, and they lie directly over the magnetic neutral line that separates the two flare ribbons. The $30 \times 10^{6} \mathrm{~K}$ feature is distinctly displaced, which we interpret to mean that it is some $35000 \mathrm{~km}$ above the other sources, since our line-of-sight is $30^{\circ}$ from the normal to the Sun's surface. Although measured in X-rays, this is very similar to the result for the Fe XxIV EUV source that Widing (1975) described earlier in this symposium. 


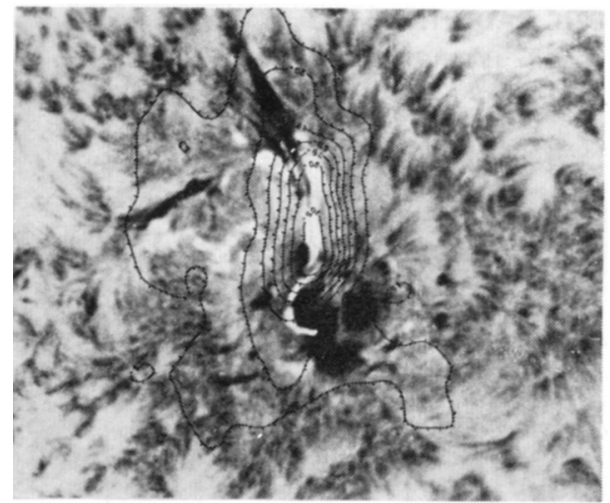

(a)

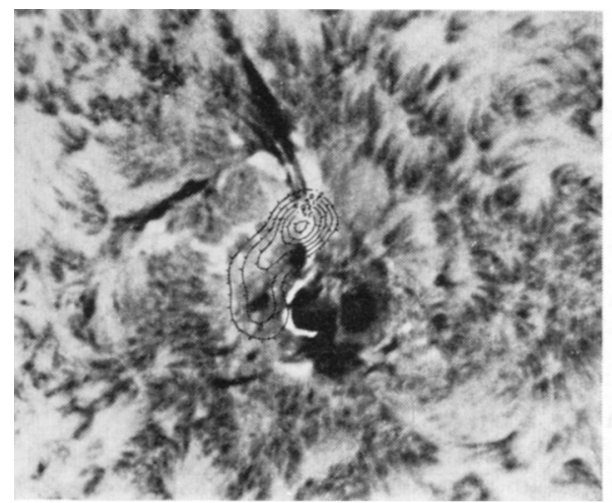

(c)

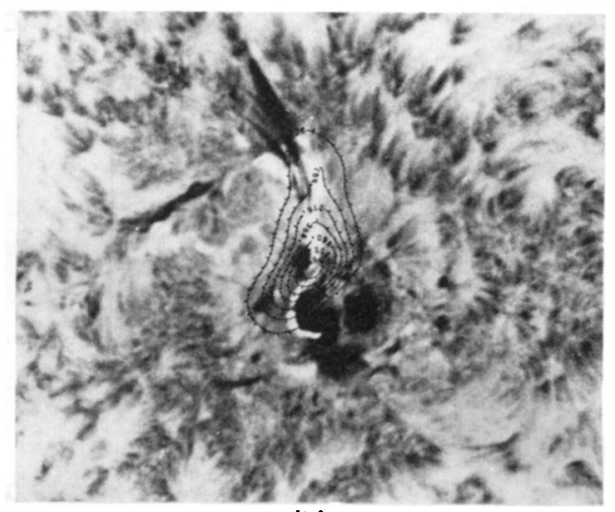

(b)

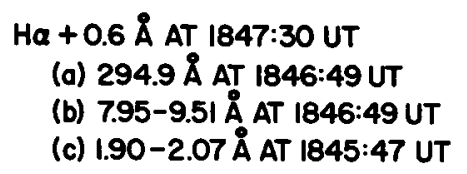

$\mathrm{Ha}+0.6 \AA$ AT 1847:30 UT

(a) $294.9 \AA$ AT 1846:49 UT

(b) $7.95-9.51$ A AT 1846:49 UT

(c) 1.90-2.07 ̊ AT 1845:47 UT

Fig. 8. Comparison of XUV spatial distribution during the gradual phase of a flare on 1972, August 2 with an off-band $\mathrm{H} \alpha$ photograph made at the Lockheed Solar Observatory. The XUV emissions are: (a) chromospheric, (b) $\mathrm{Mg} \mathrm{XI-XII}$ formed at about $9 \times 10^{6} \mathrm{~K}$, and (c) X-ray continuum characteristic of around $30 \times 10^{6} \mathrm{~K}$.

As in the active region studies, our flare observations are consistent with the coronal structure being a nested set of arches or loops connecting regions of opposite magnetic polarity. But in the flare case, the greatest temperature is found at the top of the highest arch system, not immediately above the chromosphere where the fast electrons are thought to be heating the material.

The $30 \times 10^{6} \mathrm{~K} \mathrm{Fe}$ xxv feature was observed to cool to $10 \times 10^{6} \mathrm{~K}$ in about 10 minute's time, but it is easy to show that radiation cooling was negligible during that period (unlike the case for a non-flaring active region discussed above). In fact, based on a simple model suggested by Culhane et al. (1970), conduction cooling along a magnetic arch is such an efficient process in this type of flare that the feature should have cooled about four times faster than it actually did. This means either that the hot plasma was trapped in a magnetic bottle which effectively isolated it from the chromosphere, or else that energy was being continuously supplied to the region throughout its decay. 
I have been describing some rather indirect evidence for coronal loop structures from observations made on the disk. Much more obvious are our EUV and X-ray observations of post-flare loops on the limb, shown in Figure 9. In a sense, this combines results that had been made from several different experiments on ATM.

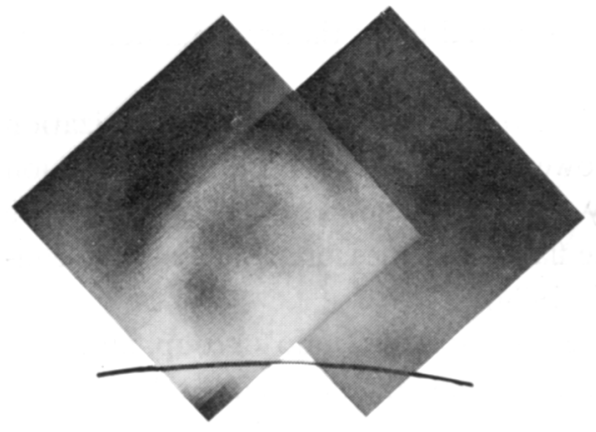

Mg IX

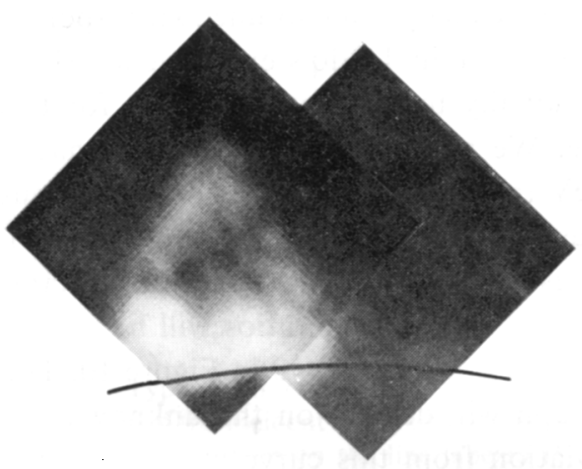

Fe XVII

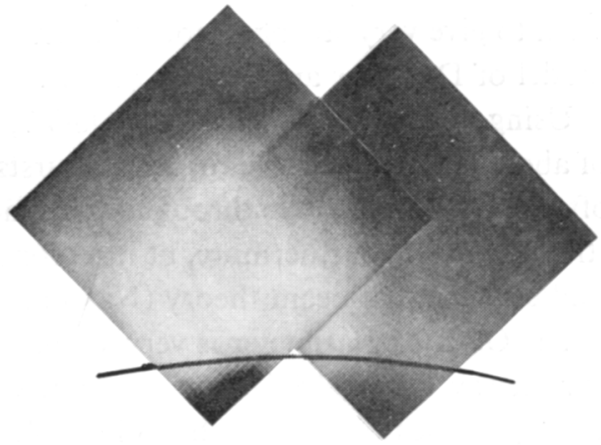

Fe XVI

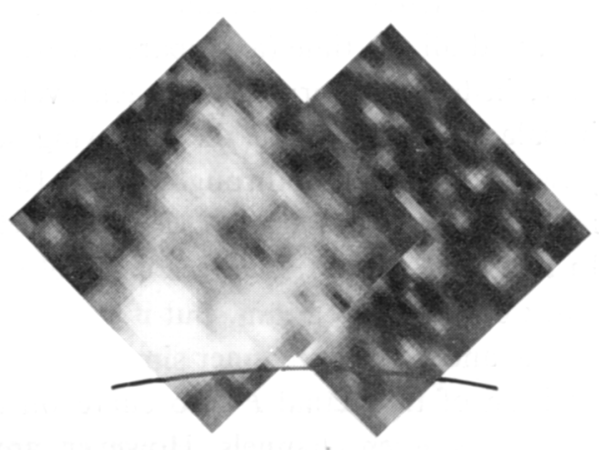

Si XIII

Fig. 9. EUV and X-ray observations from OSO 7 of post-flare loops on the south-east limb at around 0200 UT on 1972, February 9. Each image is a composite of two maps with $5 \times 5^{\prime}$ fields of view. The approximate position of the limb is indicated.

The appearance in $\mathrm{Mg}$ IX at $0.9 \times 10^{6} \mathrm{~K}$ is very similar to the classical $\mathrm{H} \alpha$ loop system, while in Fe XVI at $2.3 \times 10^{6} \mathrm{~K}$ the EUV emission is diffuse and almost featureless. In $\mathrm{X}$-rays, at even higher temperatures, the loop structure reappears but with a distinctly different shape. Furthermore, the top of the X-ray loop is particularly enhanced, as is at least one footpoint. These $\mathrm{X}$-ray emitting postflare loops are clearly not isothermal.

\section{X-Ray Polarization}

It has been recognized for some time that $\mathrm{X}$-ray polarization measurements could provide direct evidence for non-thermal processes in flares, as pointed out by Korchak 
(1967) and Elwert (1968). Recently, Tindo et al. (1970, 1972a) have reported positive detection of polarization in several X-ray bursts. By normalizing their measurements to an assumed zero polarization in the decay phase, they conclude that the impulsive component has a polarization of $10-30 \%$, although their latest paper (Tindo et al., $1972 b$ ) indicates that this level may also persist into the flare's decay. Their results seem to give very strong support in general to the radial-beam theory suggested by the model of De Jager and Kundu (1963).

Using a small polarimeter on OSO 7, we likewise have evidence for polarization of about $20 \%$ in a number of $\mathrm{X}$-ray bursts. However, we have found that polarization often seems to continue throughout the decay phase, sometimes even appearing to be strongest then. Furthermore, at least for some flares, our measurements seem to contradict the radial-beam theory (Nakada et al., 1974).

The OSO 7 polarimeter is very similar to the one used by Tindo's group, although our energy range $(15-30 \mathrm{keV})$ is somewhat higher than theirs. Incident $\mathrm{X}$-rays are Thompson scattered by a beryllium block into the surrounding three pairs of detectors. If the beam is polarized, the scattering is asymmetric, and the response of the three channels is unequal.

Unfortunately, the channels were not balanced exactly, and so unequal responses occurred all the time (as apparently was also the case in Tindo's experiment). However, instead of normalizing each event individually to attempt to correct for this imbalance, we adopted the following scheme. We assumed that the X-rays had a power-law spectrum throughout the $15-30 \mathrm{keV}$ range of our instrument. Numerous measurements of solar bursts indicate this is reasonable (e.g., Frost, 1969; Kane, 1969). In that case, the ratios of the channel responses will vary depending on the spectral index of the X-ray beam. But if the beam is unpolarized, those ratios will be related to one another in a manner similar to the solid curve labeled $P=0$ in Figure 10 . The position of the actual $P$-zero curve on the graph will depend on the unknown imbalance between channels. However, any deviation from this curve will occur only if the beam is polarized, and then will depend on the amount of the polarization and its position angle, as shown by the three closed curves in Figure 10. The dashed lines show the amount of deviation from the $P$-zero curve that would indicate polarization of at least $20 \%$.

The results using this method for eight different flares in June 1972 are displayed in Figure 11, which gives the observed ratios of channel count rates averaged over one or two minute intervals and with background subtracted. The flares occurred close enough in time that any possible drift in the imbalance between channels could not affect the results significantly. Error bars are given unless they were smaller than $4 \%$. We assumed that the $P$-zero line could be estimated by a least-squares fit through the plotted points. Any other line would only serve to increase the total amount of polarization inferred. As before, the dashed lines on the graph indicate positions that would imply polarization of at least $20 \%$.

To the lower right of Figure 11, the location of each event on the solar disk is shown, with north at the top. The cross-hatched areas are the locations on the disk 


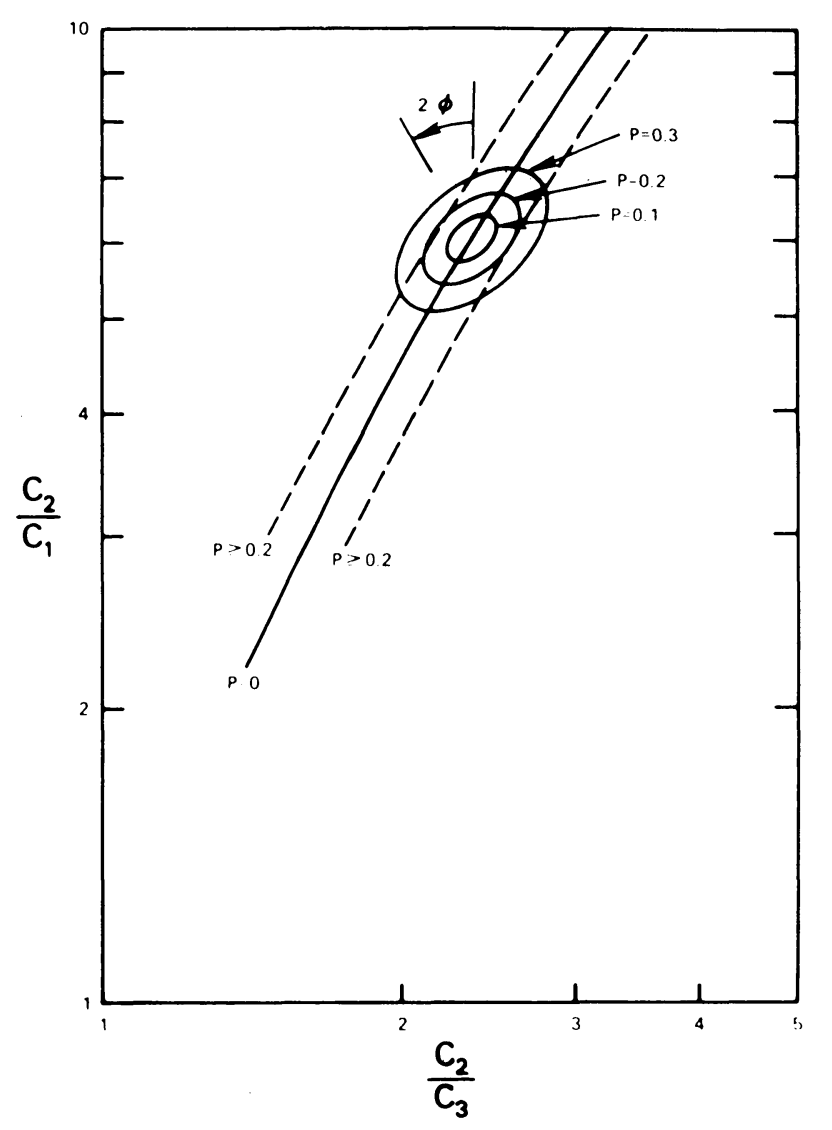

Fig. 10. Typical curves relating the ratios of counting rates for the three channels of the OSO 7 polarimeter if the incident X-rays have power-law spectra. For zero polarization, the observed ratios should fall somewhere on the line labeled $P=0$ depending on the steepness of the power law. The closed curves show deviations from a given point on the $P$-zero curve for various strengths of polarization and values of the position angle.

where the radial-beam theory predicts polarization position angles such that deviations would be along the $P$-zero line. Thus, if this theory were correct, flares such as the one on June 17 should have ratios that lie almost directly on the $P$-zero line regardless of their level of intrinsic polarization. Yet this particular flare actually exhibited the largest displacement we have yet measured. On the other hand, a limb flare outside the hatched area, such as the June 11 event, should have a very large displacement according to the radial-beam theory, whereas in fact we find it to have almost none. For these two flares at least, the belief by Tindo et al. (1972b) that polarization measurements are consistent with the radial-beam theory does not appear to be supported.

Our results for the series of great flares in August 1972 are similar in that we again measure displacements during several flares that imply a polarization of about $20 \%$. 


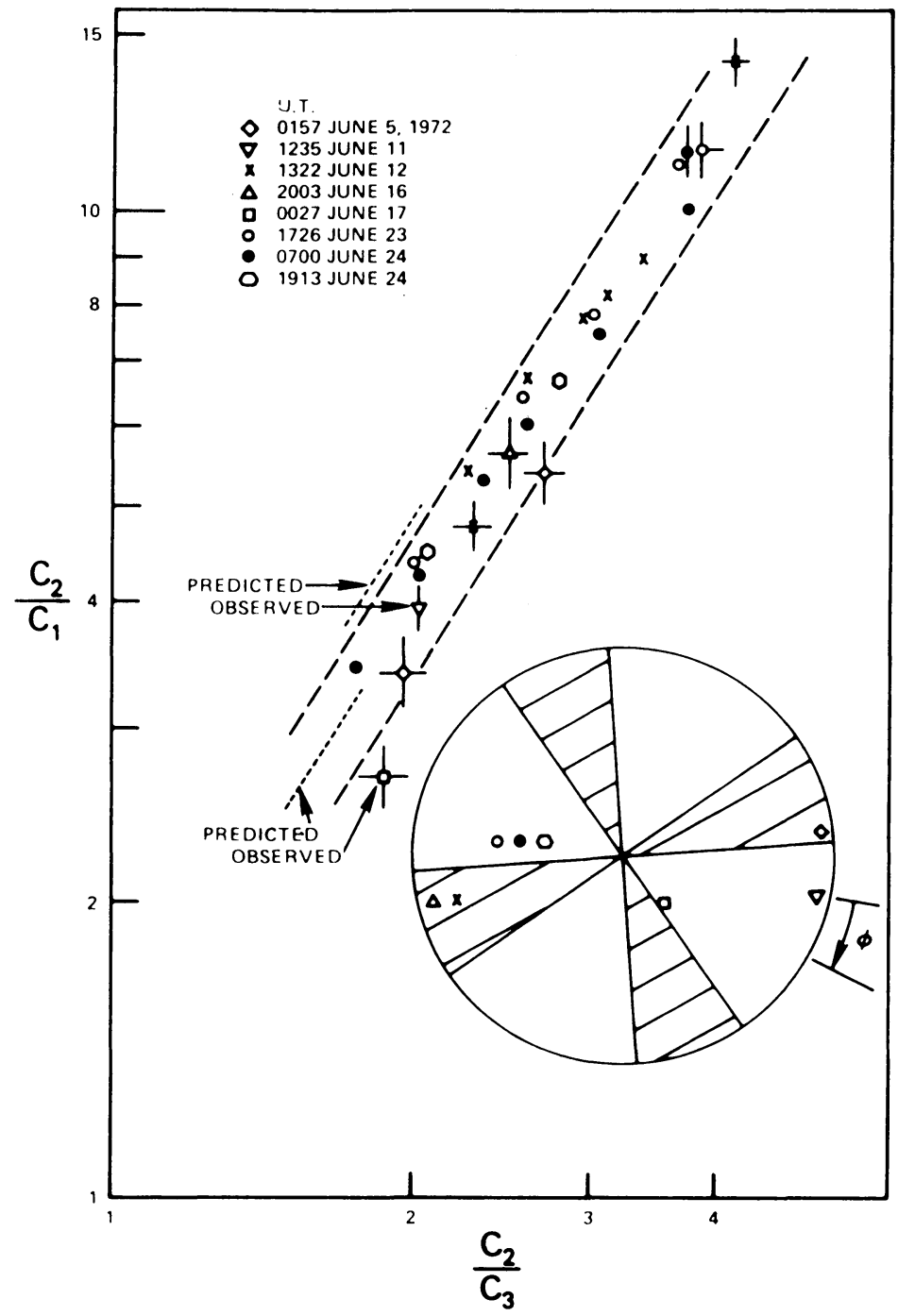

Fig. 11. Observed values of ratios of counting rates for eight X-ray events during June 1972. Standard deviations less than $4 \%$ of ratios are not shown. The dashed curves correspond to inferred polarizations of at least $20 \%$. In the lower right, the locations of the events on the solar disk are indicated, with north at the top. Figures 10 and 11 are taken from Nakada et al. (1974).

We had a particularly complete coverage of the August 7 flare, which occurred near disk center but outside of a hatched area. Figure 12 gives the time history of this $\mathrm{X}$-ray burst, along with the inferred polarization limits throughout the event. Surprisingly, the polarization appears smallest during an impulsive burst at 0350 UT, and becomes steadily stronger in the gradual phase and as the flare emission declines.

These results are exactly opposite to what I would have expected. Notice that if we had normalized this flare to an assumed zero polarization at its decay phase in the 


\section{OSO-7 POLARIMETER MEASUREMENTS}

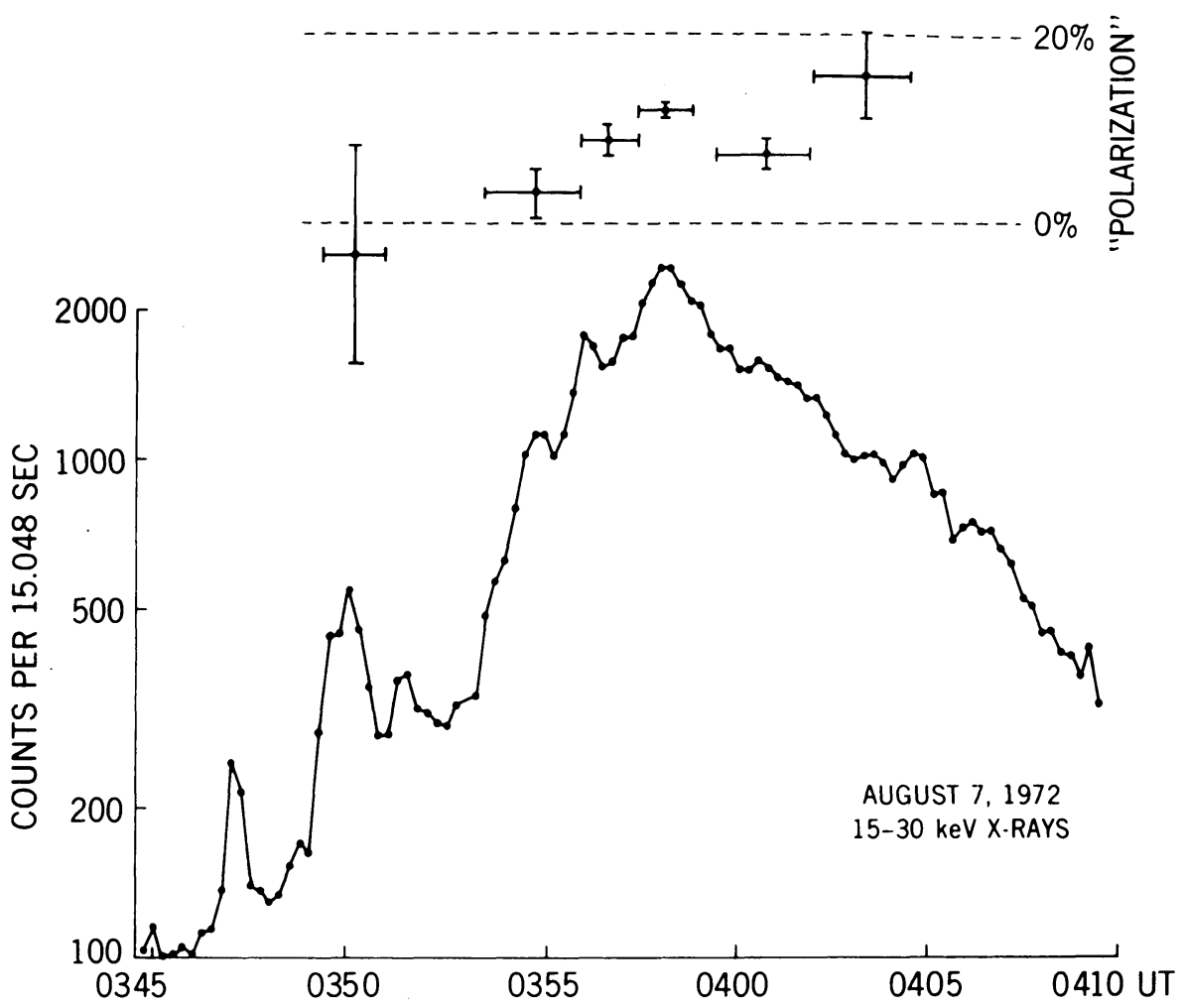

Fig. 12. Time history of the inferred polarization limits for an event on 1972, August 7 along with the X-ray flux profile for the burst. Note that the polarization may be smallest during an impulsive spike at 0350 UT, but seems definitely greater than $10 \%$ well into the decay of the gradual phase.

way that Tindo et al. (1970, 1972a) did for their measurements, the strongest polarization would then appear in the impulsive burst, just as the Tindo group reported. But if that were true, it would require that other flares in August 1972 had polarizations exceeding $40 \%$ in their declining phases.

It is of course possible that the impulsive burst did have strong polarization, but with a position angle that made our technique unable to detect it. In any case, if our reduction scheme is correct, one is forced to conclude that X-ray polarization of about $20 \%$ must exist during the decay phase of at least some flares. This might indicate that non-thermal acceleration processes are going on more or less continuously throughout the gradual component of these bursts. On the other hand, Angel (1969) has pointed out that even a thermal source can provide polarized emission if it is highly asymmetric as our observations certainly show the flare sources to be. Another possibility is that radiation backscattered from the photosphere could give rise to X-ray polarization as noted by Brown et al. (1974), although Beigman (1974) claims 
that this effect can only account for a few percent of the measured value. Perhaps the solution is actually some combination of these factors.

However it turns out, I am sure there will be many other surprises before we finally understand these strange animals called solar flares.

\section{Acknowledgments}

Parts of the research described above were originally carried out by W. M. Neupert, M. P. Nakada, and R. D. Chapman. I very much appreciate their suggestions during the preparation of this paper.

\section{References}

Angel, J. R. P.: 1969, Astrophys. J. 158, 219.

Beigman, I. L.: 1974, Preprint No. 68, Lebedev Physical Institute, Moscow.

Blake, R. L., Chubb, T. A., Friedman, H., and Unzicker, A. E.: 1964, Space Res. IV, 785.

Brown, J. C., McClymont, A. N., and McLean, I. S.: 1974, Nature 247, 448.

Chupp, E. L., Forrest, D. J., and Suri, A. N.: 1973, in R. Ramaty and R. G. Stone (eds.), High Energy Phenomena on the Sun, NASA SP-342, p. 285.

Cox, D. P. and Tucker, W. H.: 1969, Astrophys. J. 157, 1157.

Culhane, J. L., Vesecky, J. F., and Phillips, K. J. H.: 1970, Solar Phys. 15, 394.

Datlowe, D. W. and Hudson, H. S.: 1975, This volume, p. 209.

De Jager, C.: 1965, Ann. Astrophys. 28, 125.

De Jager, C. and Kundu, M. R.: 1963, Space Res. III, 836.

Donnelly, R. F.: 1967, J. Geophys. Res. 72, 5247.

Donnelly, R. F.: 1969, Astrophys. J. 158, L165.

Donnelly, R. F., Wood, A. T., and Noyes, R. W.: 1973, Solar Phys. 29, 107.

Elwert, G.: 1968, in K. O. Kiepenheuer (ed.), 'Structure and Development of Solar Active Regions', IAU Symp. 35, 444.

Frost, K. J.: 1969, Astrophys. J. 158, L159.

Jordan, C.: 1975, This volume, p. 109.

Kahler, S. W., Meekins, J. F., Kreplin, R. W., and Bowyer, C. S. : 1970, Astrophys. J. 162, 293.

Kane, S. R.: 1969, Astrophys. J. 157, L139.

Kane, S. R. and Donnelly, R. F.: 1971, Astrophys. J. 164, 151.

Korchak, A. A.: 1967, Soviet Phys. - Dokl. 12, 192.

Nakada, M. P., Neupert, W. M., and Thomas, R. J.: 1974, Solar Phys. 37, 429.

Nakagawa, Y.: 1974, Private Communication.

Neupert, W. M., Thomas, R. J., and Chapman, R. D.: 1974, Solar Phys. 34, 349.

Noyes, R. W., Withbroe, G. L., and Kirshner, R. P.: 1970, Solar Phys. 11, 388.

Pounds, K. A. and Russell, P. C.: 1966, Space Res. VII, 38.

Reidy, W. P., Vaiana, G. S., Zehnpfennig, T, and Giacconi, R.: 1968, Astrophys. J. 151, 333.

Rust, D. M.: 1974, Private communication.

Tandberg-Hanssen, E.: 1973, Earth Extraterrestrial Sci. 2, 89.

Tindo, I. P., Ivanov, V. D., Mandelstam, S. L., and Shuryghin, A. I.: 1970, Solar Phys. 14, 204.

Tindo, I. P., Ivanov, V. D., Mandelstam, S. L., and Shuryghin, A. I.: 1972a, Solar Phys. 24, 429.

Tindo, I. P., Ivanov, V. D., Valnicek, B., and Livshits, M. A.: 1972b, Solar Phys. 27, 426.

Tucker, W. H.: 1973, Astrophys. J. 186, 285.

Underwood, J. H. and Neupert, W. M.: 1974, Solar Phys. 35, 241.

Vaiana, G. S., Reidy, W. P., Zehnpfennig, T., Van Speybroeck, L., and Giacconi, R.: 1968, Science $161,564$.

Vorpahl, J. and Zirin, H.: 1970, Solar Phys. 11, 285.

Widing, K. G.: 1975, This volume, p. 153. 